EPJ Web of Conferences 82, 01030 (2015)

DOI: $10.1051 /$ epjconf/20158201030

(C) Owned by the authors, published by EDP Sciences, 2015

\title{
Research methods of reliability indicators of rectifier diode in tablet execution
}

\author{
Kurmangaliev Rinat ${ }^{\mathrm{a}}$ and Kravchenko Evgeny \\ Tomsk Polytechnic University, Lenina Av. 30, 634050 Tomsk, Russia
}

\begin{abstract}
A new forecast approach for the reliability of power semiconductor devices in cyclic operation on the basis of numerical analysis of nonuniform temperature fields is offered. We compared the failure rates of semiconductor power devices in real thermal regime with the thermal conductivity of the statistical data.
\end{abstract}

\section{Introduction}

The modern power semiconductor devices can be formally divided into two groups. The first group of devices used primarily for conversion of very high power includes diodes and thyristors. The second group of devices using low and mid-range power represents metal-oxide-semiconductor field effect transistor (MOSFET) and Insulated-gate bipolar transistor (IGBT).

Diode D123-500 in tablet execution is intended for use in DC and AC frequency to $500 \mathrm{~Hz}$ different power plants. Main Features: sealed metal-housing, clamping internal terminal connections, providing high resistance to cyclic loads, supplied forward and reverse polarity.

Model of low-frequency diode type D123-500 presented in Fig. 1. Areas of application: in vehicles, uncontrolled and half-controlled rectifier bridges, welding, galvanic.

The aim of this work - analysis of the failure intensities of a power semiconductor device based on the numerical simulation of unsteady non-uniform temperature field with the internal heat source in the range of operating temperatures.

\section{Analysis of thermal regime of device}

Analysis of the thermal regime typical diode in tablet executions conducted in one-dimensional setting. The transition temperature was taken see point $\mathrm{T}_{\mathrm{tr}}=190^{\circ} \mathrm{C}$. The geometry of the solution domain is shown in Fig. 2.

\footnotetext{
${ }^{a}$ Corresponding author: rinat real@rambler.ru
}

This is an Open Access article distributed under the terms of the Creative Commons Attribution License 4.0, which permits unrestricted use, distribution, and reproduction in any medium, provided the original work is properly cited. 


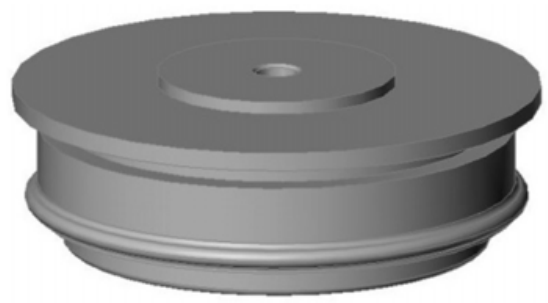

Figure 1. Rectifier Diode type D123-500 in tablet execution.

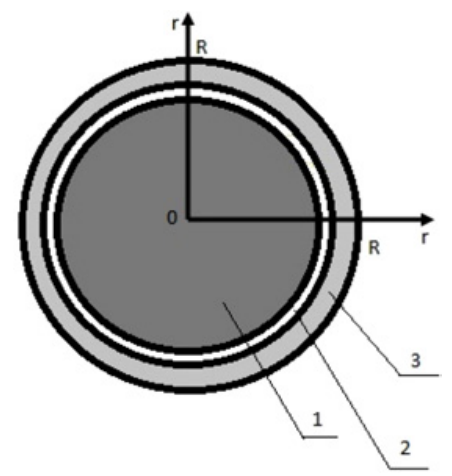

Figure 2. The geometry of the field solution (1,2,3 - areas with a different thermophysical characteristics).

The mathematical solution of the problem would be:

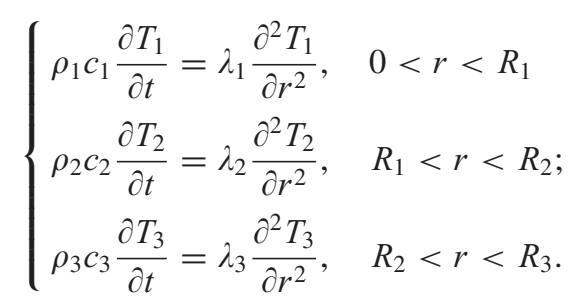

Initial and boundary conditions can be written as follows:

$$
\begin{array}{ll}
t=0: T=T_{0}, 0 \leq r \leq R ; \\
r=0: \frac{\partial T}{\partial r}=0, t>0 ; & -\left.\lambda_{1} \frac{\partial T_{1}}{\partial r}\right|_{r=R_{1}}=-\left.\lambda_{2} \frac{\partial T_{2}}{\partial r}\right|_{r=R_{1}}, \\
r=R: T=T_{h}, t>0 ; & T_{2}\left(t, R_{2}\right)=T_{3}\left(t, R_{2}\right), \\
T_{1}\left(t, R_{1}\right)=T_{2}\left(t, R_{1}\right), & -\left.\lambda_{2} \frac{\partial T_{2}}{\partial r}\right|_{r=R_{2}}=-\left.\lambda_{3} \frac{\partial T_{3}}{\partial r}\right|_{r=R_{2}} .
\end{array}
$$

Numerical simulation of the temperature field in a non-uniform circular plate with dimensions on the $\mathrm{X}$-axis equal to $\mathrm{R} 1, \mathrm{R} 2, \mathrm{R} 3$.

It was assumed that the model includes areas with different thermophysical characteristics (Table 1). In an area of radius R1 (Fig. 2) heating of the material occurred. On the boundary $r=R$ of the boundary conditions of the first kind (prescribed temperature), the point of contact 1 and 2 environments, as well as 2 and 3 environments were considered conditions the fourth kind (reflect the equality of temperatures and heat fluxes passing through the contact surface of two bodies) [1]. 
Table 1. Thermophysical properties of model elements.

\begin{tabular}{|l|l|l|l|}
\hline Material & $\lambda, \mathrm{W} / \mathrm{m}^{*} \mathrm{~K}$ & $\mathrm{C}, \mathrm{kJ} / \mathrm{kg}^{*} \mathrm{~K}$ & $\rho, \mathrm{kg} / \mathrm{m} 3$ \\
\hline Silicon & 149 & 714 & 2330 \\
\hline Aluminum & 210 & 903 & 2700 \\
\hline Ceramics & 1,8 & 680 & 1800 \\
\hline
\end{tabular}

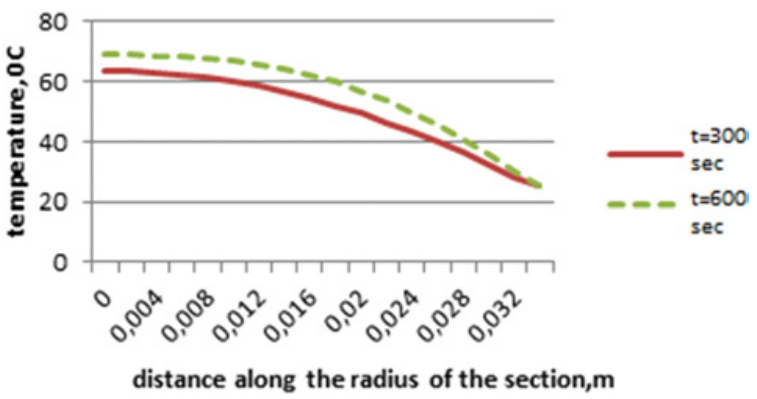

Figure 3. Distribution of temperature along the radius of the cross section.

The main assumptions used in the formulation of the problem:

1) The thermal characteristics of the materials does not depend on temperature.

2) Thermal contact at the boundaries between the areas $(1,2)$ and $(2,3)$ is considered ideal.

3) A characteristic distribution of temperature of the simulated object (diode) at ambient temperature $\mathrm{T}=25^{\circ} \mathrm{C}$ at time $\mathrm{t} 1=300 \mathrm{sec}, \mathrm{t} 2=600 \mathrm{sec}$ shown in Fig. 3 [2].

\section{Method of numerical solution}

The thermal conductivity Eq. (2) with appropriate initial and boundary conditions is solved by the finite difference method [5-7]. The diagram of splitting by coordinates was applied for the solution of difference analogues of a three-dimensional equation.

\section{Forecasting the indicators reliability of the power semiconductor device}

To analyze the reliability indicators of the diode selected mathematical models - Arrhenius and multiplicative model [3].

Multiplicative mathematical models for evaluating the reliability of the diode:

$$
\lambda_{e}=\lambda_{b f r} \cdot K_{m} \cdot K_{d n} \cdot K_{f} \cdot K_{s 1} \cdot K_{q} \cdot K_{e}
$$

where: $\lambda_{b f r}$ - base failure rate of the power device; $K_{m}$ - rate mode depending on the electric load and temperature; $K_{d n}$ - value of rate depending on the maximum set in the specifications, the electrical load; $K_{f}$ - rate of functional specificity of the operating mode of the device; $K_{s 1}$ - value of the rate as a function of the operating voltage relatively the ximum allowed by specifications; $K_{q}$ - rate of level of quality of the device; $K_{e}$ - rate of severity of service conditions.

Arrhenius model for evaluating the reliability of the diode:

$$
\lambda_{A}(T)=C \cdot \exp \left(\frac{-E}{k T}\right)
$$




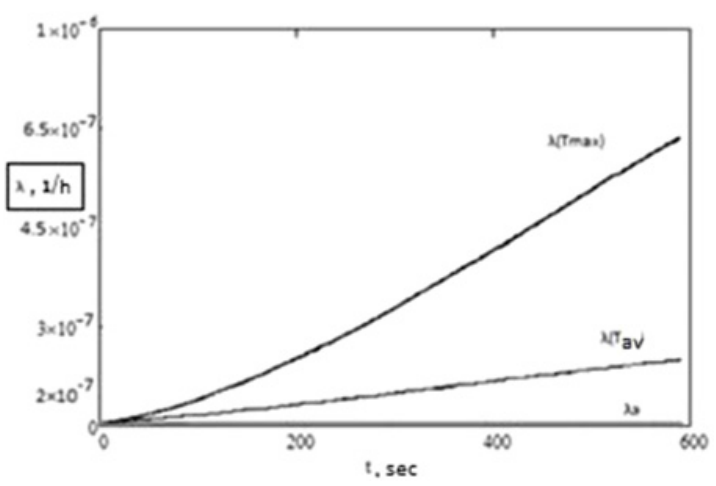

Figure 4. Failure rate of device at an ambient temperature $\mathrm{T}=25^{\circ} \mathrm{C}, \mathrm{t}=600 \sec (\lambda \mathrm{e}$ - multiplicative model (3); $\lambda\left(\mathrm{T}_{\mathrm{cp}}\right)$ - Arrhenius model (with $\left.T_{\mathrm{cp}}\right) ; \lambda\left(\mathrm{T}_{\max }\right)$ - Arrhenius model (at $\left.\mathrm{T}_{\max }\right)$ ).

where: $\mathrm{C}$ - constant, $\mathrm{E}$ - is the activation energy, $\mathrm{k}$ - Boltzmann constant.

The numerical values of $\lambda_{\mathrm{A}}\left(\mathrm{T}_{\max }\right)$, calculated according to the Arrhenius model (4), 4.5 times higher than those obtained by the multiplicative model (3) for running time $600 \mathrm{sec}$. And the ambient temperature of $25^{\circ} \mathrm{C}$. The intensity ratio of denials by the Arrhenius model $\lambda_{\mathrm{A}}\left(\mathrm{T}_{\max }\right)$ and $\lambda_{\mathrm{A}}\left(\mathrm{T}_{\mathrm{cp}}\right)$ was 2 ceteris paribus.

\section{Conclusion}

Using the multiplicative model (3) in the estimates of the failure rate of devices leads to a significant overestimation of the service life of devices $\left(\lambda_{e}=1.447 * 10^{-7}, 1 / \mathrm{h}\right)$ [4].

Prediction of reliability indices of device should be carried out on the basis of analysis of the real non-stationary non-uniform thermal regime of the device.

The work was supported by the Russian Scientific Fund (project № 14-39-00003).

\section{References}

[1] G.V. Kuznetsov, M.A. Sheremet, Differents methods for solving problems of heat conduction: a tutorial. / GV Kuznetsov, MA Sheremet. - Tomsk: Publishing House of the TPU (2007)

[2] G.V. Kuznetsov, E.V. Kravchenko, Analysis of the degradation of the polymer material of electronic devices in a spatial inhomogeneity of temperature fields // Electromagnetic waves and electronic systems (2014) №3. P. 4-12

[3] Reference "Reliability electrical radio" / psychology. S.F. Prytkov, V.M. Gorbachev

[4] GOST 27.301-95 Reliability in the art. Calculation of reliability. Basics.

[5] G.V. Kuznetsov, E.V. Kravchenko, Elektromagnitnye Volny i Elektronnye Systemy, 10 (11-12) (2005)

[6] G.V. Kuznetsov, E.V. Kravchenko, Journal of Engineering Physics and Thermophysics, 80 (5) (2007)

[7] E.V. Kravchenko, G.V. Kuznetsov, EPJ Web of Conferences, 76 (2014) 